\title{
Bemerkungen zu Gaston Bachelards Idee einer Psychoanalyse der Erkenntnis
}

Gaston Bachelard, der französische Wissenschaftsphilosoph, Poetologe der literarischen Imagination und Begleiter der Bildenden Künste seiner Zeit, hat zwei Bücher geschrieben, in deren Titel der Begriff der Psychoanalyse vorkommt. Beide erschienen 1938, zuerst „La Formation de l'esprit scientifique“ mit dem Untertitel „Contribution à une psychanalyse de la connaissance objective“ (dt.: „Die Bildung des wissenschaftlichen Geistes. Beitrag zu einer Psychoanalyse der objektiven Erkenntnis“, hier Bachelard 1987), und im November des gleichen Jahres dann ein schmales Bändchen mit dem Titel „La Psychanalyse du feu“ (dt.: „Psychoanalyse des Feuers“, hier Bachelard 1990). Beide Titel überraschen: Sie versprechen weniger die Psychoanalyse eines Subjekts als vielmehr die eines Gegenstandes. Und die Gegenstände wiederum sind so verschieden wie die objektive Erkenntnis im einen und die Imaginationen des Feuers im anderen Fall. Wie schon bei einer flüchtigen Lektüre deutlich wird, verwendet Bachelard den Begriff der Psychoanalyse auf eine ziemlich idiosynkratische Weise. Gelegentlich spricht er austauschbar auch statt von Psychoanalyse einfach von einer „Psychologie“ (Bachelard 1987, 344) oder sogar von einer „Pädagogik der objektiven Einstellung“ (ebd., 349). Ebenso erscheinen seine Gewährsleute eher eklektisch zusammengestellt. Der einzige Psychoanalytiker im engeren Sinne, der im Band über „Die Bildung des wissenschaftlichen Geistes“ öfter zu Wort kommt - insgesamt sechs Mal - ist Ernest Jones (ebd., 84-86, 93, 204, 264f.). Punktuell finden sich Hinweise auf den russischen Neurologen Constantin von Monakow (ebd., 53f., 352f.), auf den amerikanischen Psychologen James Baldwin (ebd., 345), den Schweizer Pfarrer und Psychologen Oskar Pfister (ebd., 221), den französischen Psychiater Pierre Janet (ebd., 339) und schließlich auch auf Sigmund Freud (ebd., 264). Letzterer taucht aber nur marginal und an einer einzigen Stelle auf.

Worauf will Bachelard hinaus? In der „Bildung des wissenschaftlichen Geistes“ geht es ihm einerseits darum, zu verstehen, wie wissenschaftliches Erkennen als eine Kulturleistung zustande kommt, und andererseits darum, zu klären, wie eine Geisteshaltung beschaffen sein muss, die wissenschaftliches Erkennen begünstigt. Wissenschaftliches Erkennen ist hier gleichbedeutend mit gegenständlichem Erkennen. „[W]ir [wollen] hier nicht die Psychologie des Ichs untersuchen“, heißt es im sechsten Kapitel der „Bildung des wissenschaftlichen Geistes“, „sondern die Irrtümer, auf die das Denken bei der Suche nach dem Objekt verfällt“ (ebd., 159). Oder um es mit einem unnachahmlichen Aphorismus von Paul Eluard zu sagen, den Bachelard der „Psychoanalyse des Feuers“ voranstellte: „Es gilt, nicht als Wirklichkeit zu sehen, was ich bin“ (Eluard zitiert nach Bachelard 1990, 5). 
Es geht mithin um so etwas wie eine „Psycho“-Analyse des Wirklichkeits-Denkens, des gegenständlichen Denkens also und vor allem, wie es das Zitat deutlich macht, der Fallen, die sich ihm stellen. Der Begriff der Analyse scheint hier erst einmal nichts weiter $\mathrm{zu}$ bedeuten als die Betonung, dass es weniger um die Beschreibung eines Zustands im Sinne einer logischen Struktur geht, sondern vielmehr um die Freilegung einer Dynamik, einer Aktivität. Aber es kommt noch eine Dimension hinzu, die, wie mir scheint, insgesamt charakteristisch ist für das epistemologische Denken von Gaston Bachelard. Es ist die normative Dimension, die für ihn mit jeder epistemologischen - im Gegensatz zu einer rein historischen - Analyse einhergeht. So bedeutet „Analyse“ hier also immer zweierlei, wie dies ja wohl auch in der klassischen Psychoanalyse der Fall ist: Zum einen handelt es sich um die Freilegung eines Mechanismus, der in der Regel den Charakter einer Hemmung trägt, zum anderen aber soll diese Freilegung so etwas wie Handlungsoptionen im Rahmen der analysierten Aktivität eröffnen. Den wissenschaftlichen Geist zu psychoanalysieren heißt für Bachelard also zugleich, ihn zu kultivieren und damit zu befördern.

Ich werde mich in diesen kurzen Ausführungen auf den epistemologischen Text, d.h. primär auf die „Bildung des wissenschaftlichen Geistes“ konzentrieren. Die „Psychoanalyse des Feuers“ gehört eher in das Genre der Poetologien und bedürfte einer eigenen Interpretation. Dennoch möchte ich mit einem längeren Zitat beginnen, das sich gleich am Anfang des ersten Kapitels der „Psychoanalyse des Feuers“ findet, da es wie kein anderes die Quintessenz der vorangehenden Veröffentlichung noch einmal zusammenfasst:

Einer der Vorteile der Psychoanalyse der objektiven Erkenntnis, wie wir sie vorschlagen, scheint uns gerade in der Untersuchung einer Schicht zu liegen, die weniger tiefgründig ist als die, in der sich die ursprünglichen Triebe entfalten. Und eben weil diese Schicht eine Zwischenschicht [im Original zone intermédiaire, Anm. HJR] darstellt, übt sie auf das klare, das wissenschaftliche Denken eine prägende Wirkung aus. Wissen und schöpferisches Tun sind Bedürfnisse, die man in sich selbst charakterisieren kann, ohne sie notwendigerweise mit dem Willen zur Macht in Verbindung zu bringen. Im Menschen gibt es einen regelrechten Willen zur Intellektualität. Man unterschätzt das Bedürfnis nach Verstehen, wenn man es wie der Pragmatismus und der Bergsonismus absolut dem Nützlichkeitsprinzip unterstellt. Wir schlagen also vor, unter dem Namen Prometheuskomplex all diejenigen Strebungen zusammenzufassen, die uns dazu drängen, ebensoviel zu wissen wie unsere Väter, mehr zu wissen als unsere Väter, ebensoviel wie unsere Lehrer, mehr als unsere Lehrer. Aber nur durch den Umgang mit dem Objekt, mit der Vervollständigung unserer objektiven [im schlichten Sinne von: gegenständlichen, auf Gegenstände der Welt gerichteten, Anm. HJR] Erkenntnis können wir hoffen, uns besser auf dem intellektuellen Niveau zu bewegen, das wir an unseren Eltern und unseren Lehrern bewundert haben (Bachelard 1990, 19f.).

Dieses Zitat hat es in sich, und ich werde im Verlauf meiner Ausführungen auf einige seiner Aspekte zurückkommen. Aber festzuhalten ist zunächst einmal, dass Bachelard das Erkennen, die Erkenntnistätigkeit als eine „psychologische Aktivität“ (Bachelard 1987, 83) sui generis auffasst, als eine Tätigkeit, die fortwährend eine 
Trennlinie zieht zwischen Innen und Außen, wie es am Ende des Buches über „Die Bildung des wissenschaftlichen Geistes“ heißt:

Den Augenblick der Objektivität immer zu erleben, ständig im Entstehungszustand der Objektivierung verharren, das erfordert eine unablässige Bemühung um Entsubjektivierung. Die größte Lust liegt darin, zwischen Extroversion und Introversion zu pendeln in einem Geist, der durch die Psychoanalyse von der Knechtschaft des Subjekts und des Objekts befreit ist (ebd., 357).

Der zentrale Begriff, in dem sich wie in einem Brennpunkt diese Spannung im Erkenntnisvorgang zum Ausdruck bringt, ist der von Bachelard in diesem Zusammenhang eingeführte Begriff des obstacle épistémologique, des „epistemologischen Hindernisses“ (ebd., 46-58). So heißt es gleich einleitend im ersten Kapitel - und es lohnt sich, diesen Paragraphen in seiner ganzen Länge zu zitieren:

Sucht man nach den psychologischen Voraussetzungen des wissenschaftlichen Fortschritts, so gelangt man bald zu der Überzeugung, dass das Problem der wissenschaftlichen Erkenntnis unter dem Begriff des Hindernisses angegangen werden muss. Und dabei geht es nicht um eine Betrachtung äußerer Hindernisse wie der Komplexität und Flüchtigkeit der Erscheinungen, auch nicht um eine Klage über die Schwäche der Sinne und des menschlichen Geistes: im Erkenntnisakt selbst, in seinem Innersten, erscheinen - aufgrund einer Art funktioneller Notwendigkeit - Trägheit und Verwirrung. Dort werden wir Ursachen für Stagnation und sogar Regression aufzeigen, dort die Trägheitsursachen aufdecken, die wir Erkenntnishindernisse nennen werden. Die Erkenntnis des Wirklichen ist ein Licht, das immer auch Schatten wirft. Sie ist niemals unmittelbar und vollständig. Die Enthüllung des Wirklichen ist immer rückwarts gewandt. Das Wirkliche ist niemals das, „was man glauben könnte“, es ist immer, was man hätte denken müssen (ebd., 46).

Und Bachelard schließt: „Man erkennt gegen ein früheres Wissen, indem man schlecht gegründete Erkenntnisse zerstört und das überwindet, was im Geiste selbst sich der Vergeistigung widersetzt“ (ebd.). Bachelard spricht hier aber, trotz aller seiner pädagogischen Erfahrungen, die er auch immer wieder in die Diskussion einfließen lässt, nicht als Psychologe, und er ist sich dessen auch bewusst. „Im Übrigen können wir“, bemerkt er,

in unseren Darstellungen keine Vollständigkeit anstreben, denn, dem Charakter unseres Buches gemäß, können wir keine direkte Psychologie betreiben; unser Anspruch zielt lediglich auf eine reflexive Psychologie, wie sie sich aus Überlegungen zur Erkenntnistheorie ergibt (ebd., 203).

Gegenstand dieser „reflexiven Psychologie“ sind die Entäußerungen des Erkenntnisaktes, seine Reflexe also, so wie sie sich in der Geschichte der Wissenschaften präsentieren. Die Geschichte der Wissenschaften stellt das Material zur Verfügung, das es erlaubt, den verschiedenen Verkörperungen des epistemologischen Hindernisses nachzuspüren und seine Formen nachzuzeichnen. Für Bachelard ist klar, dass das erste und entscheidende Hindernis für eine wissenschaftliche Erfassung 
der Phänomene der Wirklichkeit ihre Alltagsapprehension durch uns darstellt. Die unmittelbar sinnliche Wahrnehmung der Welt um uns herum und ihre - meist vitalistische und substanzialistische - Aufladung mit unseren Körpererfahrungen und den Formen unserer Sinneswahrnehmungen bilden das alles entscheidende Hindernis, das einer phänomengerechten Erkenntnis der Wirklichkeit entgegensteht. Der wissenschaftliche Geist entwickelt sich gegen die „ursprüngliche Erfahrung“, gegen die „anfängliche Beobachtung“. Er geht nicht aus dieser hervor, indem er sie allmählich verfeinert, sondern er muss vielmehr mit ihr brechen. Der mit dem „epistemologischen Hindernis“ korrelierende Begriff ist also der des „epistemologischen Bruchs“:

Nach unserer Ansicht müssen wir für die Epistemologie folgendes Postulat gelten lassen: das Objekt lässt sich nicht als etwas unmittelbar „Objektives“ bezeichnen; anders gesagt, ein Marsch aufs Objekt ist nicht von vornherein objektiv. Wir müssen also einen wirklichen Bruch zwischen der sinnlichen und der wissenschaftlichen Erkenntnis hinnehmen (ebd., 345).

Und dieser Bruch materialisiert sich in der Geschichte der Wissenschaften als der Übergang von der Beobachtung zum Experiment: „[Z]wischen Beobachtung und Erfahrung [besteht] nicht Kontinuität, sondern ein Bruch [...]“ (ebd., 54). Das gegenständliche Erkennen im Sinne einer wissenschaftlichen Erkenntnis lässt sich somit nicht als Besitz charakterisieren, in den ein Individuum ein für alle Mal gelangen könnte, sondern allein als ein unabschließbarer Prozess. Und als ein solcher ist er auch nicht auf die Leistung eines Individuums reduzierbar:

\footnotetext{
Um ganz sicher zu sein, dass der Reiz nicht länger die Grundlage unserer Objektivierung bildet, um ganz sicher zu gehen, dass die Gegenstandskontrolle eine Reform und kein Echo ist, muss man zu einer sozialen Kontrolle gelangen. Folglich schlagen wir vor - selbst um den Preis, dass man uns eines circulus vitiosus bezichtigen sollte - die Objektivität auf das Verhalten des anderen zu gründen; wir wollen [...] das Auge des anderen - und stets das Auge des anderen dazu ausersehen, die Form - die endlich abstrakte Form - der objektiven Erscheinung zu sehen: sage mir, was du siehst, und ich sage dir, was es ist (ebd., 346).
}

Dieser letzte Satz ist übrigens ein unüberhörbarer Anklang an Eluards Aphorismus, den ich eingangs zitierte. Bachelard fährt fort: „Allein dieser unsinnig erscheinende Zirkel vermag uns einige Sicherheit zu geben, dass wir von unseren ursprünglichen Wahrnehmungen vollständig abstrahiert haben“ (ebd.).

Bachelards historische Epistemologie ist letztlich eine Epistemologie des Irrtums, wie sie auch der italienische Mathematiker und Wissenschaftshistoriker Federigo Enriques, auf den sich Bachelard hier bezieht, in seinem Essay über „Die Bedeutung der Geschichte des wissenschaftlichen Denkens“ (Enriques 1934) entwickelt hat. Die Voraussetzung für wissenschaftliches Erkennen ist die Fähigkeit, eine alte Erkenntnis als Irrtum abzulegen. Die Struktur des Bruches setzt sich also in der Entwicklung der Wissenschaften fort und beschränkt sich nicht auf einen einmal vollzogenen Schnitt mit der Alltagserfahrung. Bachelard zögert nicht, diese Bruch- 
struktur unter den Begriff der „Psychoanalyse“ zu bringen. Anlässlich der historischen Entwicklung der Mathematik heißt es:

Ich zögere auch nicht, die Strenge als eine Psychoanalyse der Anschauung und das algebraische Denken als eine Psychoanalyse des geometrischen Denkens zu präsentieren (ebd., 342).

An anderer Stelle heißt es wiederum:

Die Psychoanalyse, die nach unserer Vorstellung in einen affektiven Bildungsprozess eingeschaltet werden sollte, muss gerade die Interessen verlagern (ebd., 42).

Der Irrtum nimmt hier die generalisierte Form einer Konzentration auf Differenz an, aber einer mit einem normativen Index versehenen Differenz:

[W]as nützt eine Erfahrung, die keinen Irrtum richtigstellt, die schlicht und ohne Debatte wahr ist. Eine wissenschaftliche Erfahrung ist also eine Erfahrung, die der gewohnten Erfahrung widerspricht. Übrigens behält die unmittelbare und gewöhnliche Erfahrung immer einen in gewisser Hinsicht tautologischen Charakter, sie entwickelt sich im Reich der Wörter und Definitionen; hier fehlt gerade diese Perspektive korrigierter Irrtümer, die in unseren Augen das wissenschaftliche Denken ausmacht (ebd., 44).

Einer bestimmten Form dieser Erfahrung oder Erklärung, die eng mit einem Bild aus der Alltagserfahrung verknüpft ist, und die in der Physik des siebzehnten und achtzehnten Jahrhunderts vorherrschend war, hat Bachelard ein ganzes Kapitel gewidmet. Es handelt vom Schwamm (l'éponge) als dem typischen Beispiel eines epistemologischen Hindernisses.

Der Schwamm, so wie wir ihn aus unseren Alltagsverrichtungen kennen und mit ihm umgehen, hat etwas sehr Augenscheinliches und empirisch unmittelbar Evidentes. Das Gebilde ist von poröser Natur, die Fasern, aus denen es besteht, hängen zwar zusammen, sparen aber ein Netz von Hohlräumen aus. Ein Schwamm ist aus diesem Grund in der Lage, andere Materien wie Gase und Flüssigkeiten in sich aufzunehmen und sich mit ihnen vollzusaugen, ohne sich mit ihnen zu vermischen. Für Descartes, so urteilt Bachelard, ist er ein Beispiel für einen „rarefizierten“ Körper, also einen Körper, dessen Kompaktheit aufgelockert ist und dessen Eigenschaft durch diese Auflockerung definiert ist:

[E]in Schwamm zeigt uns die Schwammhaftigkeit. Er zeigt uns, wie ein bestimmter Stoff sich mit einem anderen Stoff, anfülltt. Diese Lektion über die heterogene Fülle reicht aus, alles zu erklären. Descartes' Metaphysik des Raumes ist die Metaphysik des Schwammes (ebd., 135).

Man sieht an diesem Beispiel gut, warum Bachelard diese Art von epistemologischen Hindernissen, die das Alltagswissen dem wissenschaftlichen Wissen bietet, auch als „sprachliches Hindernis“ (ebd., 127) bezeichnet. Es besteht letztendlich in einer Tautologie: der Schwammhaftigkeit des Schwammes. Die Medizingeschichte der frühen Neuzeit ist voll mit Erklärungen dieser Art, aber auch in der Physik sind 
sie zahlreich, wie Bachelard uns in seinem Kapitel über den Schwamm belehrt. So zitiert er etwa das Beispiel von René Antoine de Réaumur (1683-1757), der auf diese Weise die Komprimierbarkeit der Luft erklärt: Die Luft ist ein Schwamm, nur eben einer, der noch schwammhafter ist als ein ordinärer Schwamm, mit dem man die Luft aber durchaus vergleichen und womit man ihre elastischen Eigenschaften erklären kann. Wir sehen hieran, so Bachelard, was es heißt, „ein generalisiertes Bild“ (ebd., 128) zu sein, „das durch ein einziges Wort, das Leitmotiv einer wertlosen Vorstellung, ausgedrückt wird“ (ebd.). Die wissenschaftliche Erkenntnis muss sich solcher in der Alltagsempirie geformter Bilder, die sich zu einer „Bewegung rein sprachlicher Art“ (ebd., 130) zusammenziehen, entledigen, um sie schließlich durch mathematische Relationen zu ersetzen. Solche Bilder können allerdings, so Bachelard, sekundär wieder ins Spiel kommen, um unanschaulich gewordene Beziehungen mit „ein wenig Farbe“ (ebd., 134) zu versehen und so das wissenschaftliche Denken zusätzlich zu befeuern. Ein Beispiel dafür wäre etwa, Energiepotentiale durch Hebevorgänge zu illustrieren.

Der Schwamm steht hier als historisches Beispiel für einen zentralen Begriff in Bachelards Idee einer historischen Epistemologie: das epistemologische Hindernis. Diese Epistemologie geht davon aus, dass das wissenschaftliche Wissen einen Bruch mit dem Alltagswissen und seinen konkreten Bildern vollziehen und so „der Gefahr [...] unmittelbare[r] Metaphern“ (ebd., 138) entgehen muss, um sich zu konstituieren. Der „epistemologische Bruch“ mit der Bilderwelt des Alltags ist entscheidend. Ebenso entscheidend aber ist im Verlauf der weiteren Entwicklung des wissenschaftlichen Wissens, dass der erreichte Stand des jeweiligen Wissens selbst immer wieder gewissermaßen in die Alltäglichkeit absinken und sich so jederzeit von neuem als epistemologisches Hindernis manifestieren kann. Man kann, so glaube ich, an diesem Beispiel ganz gut nachvollziehen, welche allgemeine Form die Figur des Psychoanalytischen bei Bachelard annimmt. Kurz gesagt besteht sie darin, dass eine Verschiebung, die einmal virulent war und nun nicht mehr wahrgenommen wird, wieder ins Bewusstsein gehoben und damit problematisierbar wird. Man könnte es auch so formulieren: Das wissenschaftliche Wissen, das Wissen auf dem Weg zum Gegenstand, ist ein ständiger Kampf gegen abgesunkene Metaphern. 


\section{Literaturverzeichnis}

Bachelard, Gaston (1987): Die Bildung des wissenschaftlichen Geistes. Beitrag zu einer Psychoanalyse der objektiven Erkenntnis [1938], Frankfurt a.M.

Bachelard, Gaston (1990): Psychoanalyse des Feuers [1938], Frankfurt a.M.

Enriques, Federigo (1934): Signification de l'histoire de la pensée scientifique, Paris. 
